\title{
A Patient with a "Strawberry Tongue"
}

\author{
James Guggenheimer, DDS* \\ Department of Diagnostic Sciences, University of Pittsburgh School of Dental Medicine, USA
}

\begin{abstract}
The primary constituents of saliva are water and mucins that keep the oral mucous membranes moist and lubricated. When salivary gland secretions are diminished or lost, patients may develop symptoms of oral dryness (xerostomia), burning, soreness, and difficulty with eating, taste, and swallowing. The use of xerogenic medications is one of the major causes of reduced salivary gland function. This case report describes a patient with mood disorders and fibromyalgia for which he was taking five medications that are associated with xerostomia and hyposalivation. In order to alleviate his oral dryness, he began to use a homemade beverage that discolored his tongue and masked a median rhomboid glossitis. This lesion is a manifestation of infection by Candida albicans that can develop as a consequence of a loss of saliva and its constituent protective antimicrobial proteins.
\end{abstract}

\section{Keywords}

Saliva, Hyposalivation, Drug effects, Adverse effects, Oral candidiasis, Salivary proteins and peptides

\section{Case Presentation}

A 54-year-old man presented to the dental clinic complaining of pain in his maxillary left canine. The tooth had been restored two years previously but had now developed secondary caries, fractured, and was determined to be nonrestorable. The patient's medical history included depression, chronic fatigue syndrome, fibromyalgia, gastro-esophageal reflux, and elevated cholesterol. He denied smoking. His current medications included venlafaxine $\mathrm{HCl}\left(\right.$ Effexor $\left.^{\circledR}\right)$, bupropion $\mathrm{HCl}$ (Wellbutrin ${ }^{\circledR}$ ), and aripiprazole (Abilify ${ }^{\circledR}$ ) for depression, esomeprazole (Nexium ${ }^{\circledR}$ ) for gastro-esophageal reflux, fenofibrate $\left(\right.$ Tricor $\left.^{\circledR}\right)$ for elevated cholesterol and

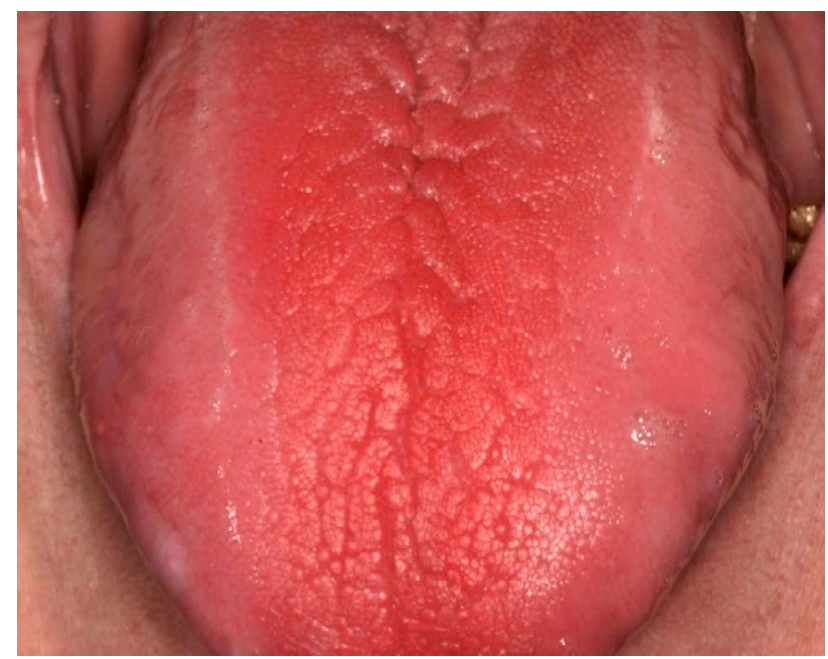

Figure 1: Initial appearance of the atrophic and fissured tongue with the intense, red coloration. naproxen sodium (Aleve ${ }^{\circledR}$ ) for chronic muscle and joint pain. The oral examination revealed a fiery red, fissured, and atrophic dorsal surface of the center of the tongue (Figure 1). The labial and buccal mucosa felt dry to palpation. At this point, the patient reported that he had symptoms of dry mouth for which he was drinking a homemade, sweetened, red colored beverage that he had brought with him (Figure 2). The patient was advised to substitute water and to suck on ice chips for his symptoms instead of drinking the sweetened beverage, and the maxillary canine was extracted. At his follow-up visit two weeks later, the dorsal surface of the tongue now revealed a fissured, atrophic, erythematous region along the midline (Figure 3). This appearance was consistent with central papillary atrophy (median rhomboid glossitis) that is one of the manifestations of erythematous candidiasis [1]. A 10-day regimen of $10 \mathrm{mg}$ clotrimazole (Mycelex ${ }^{\circledR}$ ) troches to be held in the mouth 4-5 times a day was prescribed, but the patient failed to return for his next follow-up visit.

Our final assessment was central papillary atrophy of the tongue that had been stained by a red, food-coloring additive in a homemade beverage that the patient was using to relieve his symptoms of xerostomia.

*Corresponding author: James Guggenheimer, DDS, Department of Diagnostic Sciences, University of Pittsburgh School of Dental Medicine, G-137 Salk, 3501 Terrace Street, Pittsburgh, PA 15260, USA, Tel: 412-648-8627

Accepted: April 23, 2019

Published online: April 25, 2019

Citation: Guggenheimer J (2019) A Patient with a "Strawberry Tongue". Archives Oral Maxillofac Surg 2(1):26-28

Copyright: (c) 2019 Guggenheimer J. This is an open-access article distributed under the terms of the Creative Commons Attribution License, which permits unrestricted use, distribution, and reproduction in any medium, provided the original author and source are credited. 


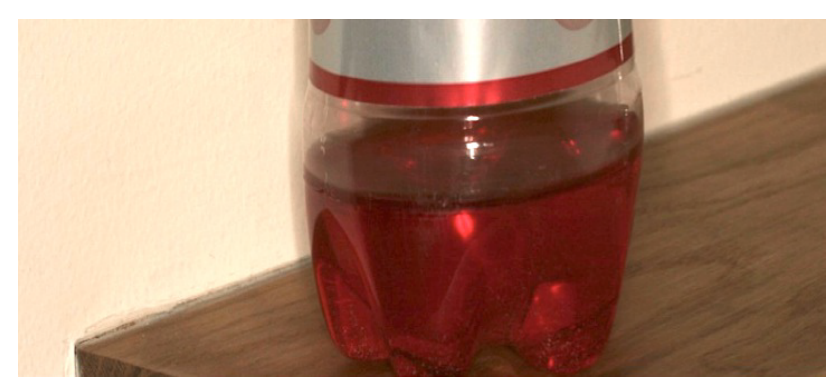

Figure 2: The patient's homemade beverage.

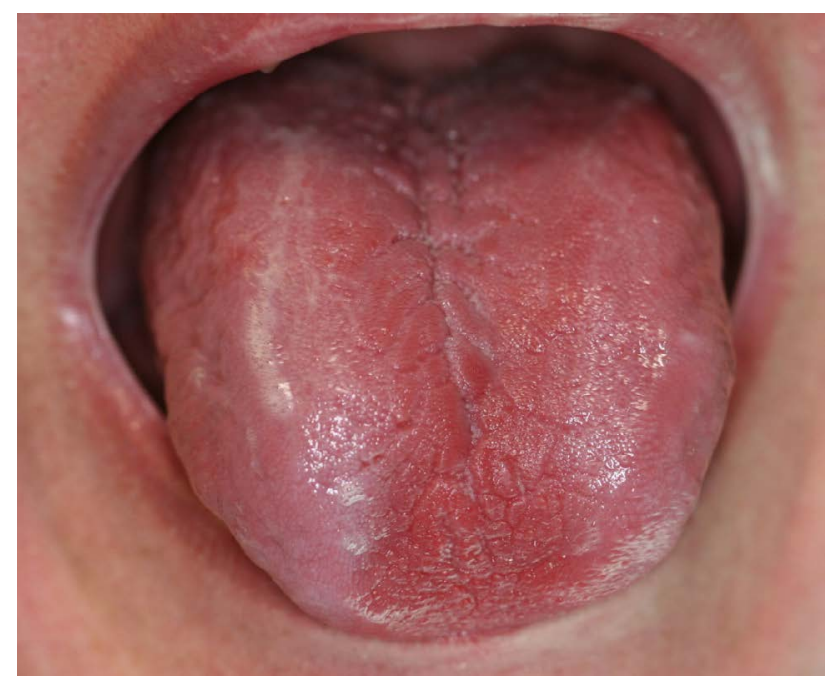

Figure 3: Appearance of the tongue two weeks after discontinuing use of the homemade beverage.

\section{Discussion}

A reduction in or loss of salivary gland function is primarily caused by the use of medications [2]. A number of drugs have been associated with this side effect, particularly if they have anticholinergic or parasympatholytic effects or modify neurotransmission such as psychotherapeutic agents or anticonvulsants $[2,3]$. Other commonly used prescription or over-the-counter-medications that have been associated with loss of saliva or symptoms of xerostomia include antihistamines, diuretics, nonsteroidal anti-inflammatory agents, and drugs for gastrointestinal and respiratory diseases [2-4]. The use of multiple medications also increases the likelihood of hyposalivation, particularly in the elderly population [5]. This is particularly relevant to data from U.S. surveys collected between 2011-2014 which found that $91 \%$ of patients 65 years and older had used at least one prescription drug, $67 \%$ had taken three or more, and $41 \%$ had taken five or more in the past 30 days [6].

A reduction in or loss of salivary gland function has also been associated with a number of systemic conditions that include autoimmune diseases such as fibromyalgia in this patient, diabetes, cirrhosis, and primary and secondary Sjögren's syndrome. Radiation therapy to the head and neck or treatment with radioactive iodine for cancer of the thyroid are additional risk factors for loss of salivary gland function
$[1,2]$.

Saliva is essential for the maintenance of oral health based on its fluid content that bathes and irrigates the oral cavity, and a large number of proteins and peptides that have antimicrobial properties for maintaining a normal balance of oral flora [7]. When there is a disruption in this balance, an overgrowth of Candida albicans can result as a consequence of loss of saliva as well as other risk factors such as cigarette smoking, use of dentures, recent use of an antibiotic, diabetes, or immunosuppression [1-3,8]. The only risk factor for candidiasis in this patient, however, was medicationinduced hyposalivation from the xerogenic side effects of the five medications that he was taking. It is also of interest that all of the medications that he was taking were among the 200 most frequently prescribed drugs in the United States in 2014 [9].

Central papillary atrophy of the tongue (median rhomboid glossitis) is a manifestation of erythematous candidiasis that develops in the midline of the dorsal surface of the tongue [1]. It is characterized by a well-circumscribed erythematous oval or rhomboid-shaped patch that is typically located at the junction of the anterior two-thirds and posterior third of the tongue [1]. "Strawberry tongue", on the other hand, is the term used to describe the appearance of the dorsal surface of the tongue four or five days after the onset of scarlet fever (scarlatina). This was first recognized in 1842 $[10,11]$ and the descriptive term "strawberry" was based on the development of erythema and hyperplasia of the fungiform papillae [1]. The development of a strawberry tongue is considered to be pathognomonic of scarlet fever [1], an infection caused by group $A, \beta$-hemolytic streptococci that primarily affects children between the ages of 3 and 12 [1]. Other manifestations of scarlet fever include sore throat, pharyngitis, fever, and a skin rash [1], none of which were apparent in our patient.

\section{References}

1. Neville BW, Damm DD, Allen CM, et al. (2015) Oral and Maxillofacial Pathology. ( $4^{\text {th }}$ edn), Elsevier, St. Louis.

2. Scully C (2003) Drug effects on salivary glands: Dry mouth. Oral Dis 9: 165-176.

3. Guggenheimer J, Moore PA (2003) Xerostomia: Etiology, recognition and treatment. J Am Dent Assoc 134: 61-69.

4. Wynn Rl, Meiller TF, Crossley HL (2016) Drug Information Handbook for Dentistry. (22 ${ }^{\text {nd }}$ edn), Lexi-Comp, Wolters Kluwer.

5. Nederfors T, Isaksson R, Mornstad H, et al. (1997) Prevalence of perceived symptoms of dry mouth in an adult Swedish population-- relation to age, sex, and pharmacotherapy. Comm Dent Oral Epidemiol 25: 211-216.

6. (2016) Health, United States, 2016, with chartbook on long-term trends in health.

7. Marsh PD, Do T, Beighton D, et al. (2016) Influence of saliva on the oral microbiota. Periodontol 2000 70: 80-92.

8. Guggenheimer J, Moore PA, Rossie K, et al. (2000) Insulin dependent diabetes mellitus and oral soft tissue pathologies. II. Prevalence and characteristics of Candida and candidal lesions. Oral Surg Oral Med Oral Pathol Oral Radiol Endod 89: 570-576. 
9. Bartholow M (2015) Top 200 Drugs of 2014. Pharmacy Times.

10. Watson (1842) Course of clinical lectures delivered at the Middlesex Hospital. Lecture VIII. December 3, 1842. Prov Med J Retrosp Med Sci 5: 223-225.
11. Haller JS (1982) The foul tongue: A 19th century index of disease. (History of Medicine). West J Med 137: 258-264.

DOI: $10.36959 / 379 / 350$ 